1.0\%. The FIT positive rate was $7.0 \%$, the adenoma detection rate was $21.8 \%$, and the CRC detection rate was $2.5 \%$. In addition, the colonoscopy compliance rate $(79.1 \%)$, rate of adequate bowel preparation (86.3\%), and the colonoscopy completion rate $(93.1 \%)$ were high. We found the pooled complication rate of $0.3 \%$, with PPVs of FIT being $39.2 \%$ (for adenoma) and $4.7 \%$ (for CRC). The range of all indicators varied substantially. In subgroup analysis, a higher FIT cut-off value indicated lower positivity and lower adenoma detection rate. Compared with CRC screening programmes with one FIT, those with two FIT had lower participation rate $(48.8 \%$ vs $55.5 \%)$ but higher positive rate $(8.9 \%$ vs $5.8 \%)$ and adenoma detection rate $(21.8 \%$ vs $17.3 \%$ ).

Conclusions Our findings reported the pooled performance indicators of different CRC screening programmes. The summary measures could inform the benchmarking of performance indicator targets across different CRC screening programmes.

\section{IDDF2020-ABS-0143 A SINGLE-CENTRE PROSPECTIVE AUDIT OF INPATIENT CARE FOR ADULTS WITH ULCERATIVE COLITIS}

${ }^{1}$ Wilson Siu*, ${ }^{1}$ Hannah Lambert, ${ }^{2}$ Jason On, ${ }^{1}$ Malcolm Smith. ${ }^{1}$ Department of Digestive Disorders, Aberdeen Royal Infirmary, UK; ${ }^{2}$ Department of Colorectal Surgery, Aberdeen Royal Infirmary, UK

\subsection{6/gutjnl-2020-IDDF.89}

Background Acute severe ulcerative colitis (UC) is a potentially life-threatening presentation and requires coordinated multidisciplinary management. The aim of our prospective audit was to evaluate the inpatient management and outcome of patients with UC.

Methods All consecutive patients admitted to Aberdeen Royal Infirmary for treatment of acute UC were prospectively recruited in the study over a 6 months period. All patients were followed up for a minimum of 3 months from the discharge date. Patient's demographics, clinical data, endoscopic assessment, medical and surgical treatment details were collected. The clinically significant outcome was defined as steroid treatment failure requiring surgery or rescue therapies (ciclosporin or infliximab) despite intravenous steroid therapy. Statistical comparisons were made using Non-parametric Mann-Whitney test and Fishers Exact test.

Results 27 patients (15 females; median age 41 years (IQR 31-63)) were admitted for treatment of UC during the 6 months period. 23 patients had severe UC as per Truelove and Witts Score. 19 patients had pre-existing diagnosis of UC prior admission. Faecal calprotectin was checked in 3 patients and were all $>1000 \mathrm{ug} / \mathrm{g}$.

All patients received a minimum of 3 days of intravenous steroid. 10 patients were non-responsive to steroid therapy. 3 patients received rescue infliximab while 1 patient received ciclosporin. 7/27 (26\%) required in-patient colectomy during the study period. Previous diagnosis of UC, previous admission or mesalazine use were not associated with steroid treatment failure. Only bloody stool frequency at Day 3 had a statistically significant association $(p=0.03)$. The median (IQR) bloody stool frequency was $5(1.8-10.3)$ for those who were in the steroid failure group compared to $3(0-3)$ for those in the steroid responder group.

Conclusions In our prospective study, the in-hospital colectomy rate was $7 / 27(26 \%)$ for acute presentation of UC. Systemic oral steroid use prior to admission was associated with steroid treatment failure but was not statistically significant $(p=0.05)$. Future study with a larger sample size could perhaps identify more clinical and laboratory variables that could be useful to stratify patients at risk of steroid failure.

\section{IDDF2020-ABS-0144 CATASTROPHIC EMERGENCY EVENTS OF COMPLICATED ULCERATIVE COLITIS: AN IMPACT ON CLINICAL OUTCOME}

${ }^{1}$ Ida Ayu Setyawati Sri Krishna Dewi ${ }^{*},{ }^{2}$ Budhi Ida Bagus. 'Medical Faculty, Pendidikan Ganesha University, Indonesia; ${ }^{2}$ Department of Surgery, Sebelas Maret University, Indonesia

\subsection{6/gutjnl-2020-IDDF.90}

Background As one of the inflammatory bowel diseases case, ulcerative colitis (UC) has many clinical manifestations, including usual clinical symptoms or emergency presentation of this part of IBD. Surgery is the last treatment option of ulcerative colitis, in the era of better medication over the last decades, we could decrease the need for elective or emergency surgeries. The two most common emergency case was uncontrolled hemorrhage and colonic perforation.

Methods We would evaluate the emergency presentation of ulcerative colitis in our institution during the last 6 months from January until June 2020. All patients with already known as ulcerative colitis will be included in this study, clinical presentation and the surgical option will be recorded. The clinical outcome will be evaluated from inpatients mortality and postoperative morbidity if present.

Results During the last 6 months, we reported 1 case of 74 years old male with diffuse peritonitis due to hollow viscous perforation, free intraperitoneal air was found on abdominal $\mathrm{x}$-rays, the patient only has diarrhea symptom before and no history of specific medication for his ulcerative colitis. This case was found incidentally on an emergency setting, and he had sepsis condition with cardiopulmonary comorbidity. Surgery has been done, and total colectomy was the option on this case with stoma creation. Cardiopulmonary support already has been given in the intensive care unit and no recovery from the prolonged sepsis after the first 24 hours. This complicated UC condition with late presentation case has an unfavorable outcome on catastrophic emergency condition.

Conclusions Ulcerative colitis has a life-threatening emergency presentation, late presentation, and preoperative comorbidities play an important role in the unfavorable outcome on complicated ulcerative colitis cases.

\section{IDDF2020-ABS-0145 COMBINATION OF ANTIBIOTICS AS ADJUVANT THERAPY IN ACUTE SEVERE ULCERATIVE COLITIS: A RANDOMIZED TRIAL}

Shubhra Mishra*, Harshal Mandavdhare, Harjeet Singh, Arup Choudhury, Jimil Shah, Sant Ram, Dimple Kalsi, Jayanta Samanta, Kaushal Prasad, Arun Sharma, Usha Dutta, Vishal Sharma. Postgraduate Institute of Medical Education and Research, India

\subsection{6/gutjnl-2020-IDDF.91}

Background Some studies have suggested that targeted combination therapy with antibiotics could improve response in active ulcerative colitis, but similar data is not available in acute severe UC (ASUC). 